\title{
Immunodominant antigens of Streptococcus equisimilis shared by other $\beta$-haemolytic streptococci
}

\author{
N. CIMOLAI* and D. G. MAH \\ Division of Medical Microbiology, Department of Pathology, University of British Columbia, Vancouver, British \\ Columbia, Canada
}

\begin{abstract}
Summary. Three immunodominant antigens of Streptococcus equisimilis (Lancefield group C) with approximate mol. wts of 46,66 and $105 \mathrm{kDa}$ were recognised by human serum IgG and IgA immunoblotting. These antigens were identified consistently by various human sera but immunoblots with IgA (heavy chain) and secretory IgA (J chain) from human respiratory secretions gave more variable results. Antigens with similar migration rates were demonstrated in S.pyogenes, large colony human biotype group G streptococci, and streptococci of groups C and G from the " $S$. anginosus-milleri group". Polyclonal antibody which was eluted from immunoblot substrates that contained the $S$. equisimilis $66-\mathrm{kDa}$ antigen reacted with the $60-\mathrm{kDa}$ antigen of $S$. pyogenes. Both polyclonal and monoclonal anti-vimentin antibodies identified the 46-kDa and 66-kDa antigens of $S$. equisimilis. The homology of these antigens among $\beta$-haemolytic streptococci has the potential to complicate both a strategy for the utilisation of immunoblotting for diagnostic purposes and the understanding of how such antigens may be involved in the pathogenesis of post-infectious sequelae.
\end{abstract}

\section{Introduction}

Although a cause-and-effect relationship between group A streptococci (Streptococcus pyogenes) and pharyngitis has been recognised for decades, a similar relationship has not been defined for most other $\beta$ haemolytic streptococci. ${ }^{1}$ In part, the study of the association of $\beta$-haemolytic non-group $\mathrm{A}$ streptococci with pharyngitis has been complicated by the diversity of species within some Lancefield groups. ${ }^{1}$ Furthermore, much of the previous work on a possible role for the $\beta$-haemolytic non-group A streptococci has not been able to benefit from the insight provided by relatively recent genetic studies that have demonstrated significant genetic homology between streptococci of different Lancefield groups. ${ }^{2,3}$

Previously, we published circumstantial evidence that supported a link between large colony streptococci of groups $\mathrm{C}$ and $\mathrm{G}$ and symptomatic pharyngitis in children. ${ }^{4}$ Later, in a case-control study, we showed that moderate to heavy quantities of $S$. equisimilis (group C) and large colony human biotype group G streptococci were associated with symptomatic pharyngitis. ${ }^{5}$ The latter findings for $S$. equisimilis have been supported recently by the work of Turner et al. ${ }^{6} \mathrm{~A}$ cause-and-effect relationship could be strengthened by various further investigations that could, conceivably,

Received 22 July 1993; revised version accepted 21 Oct. 1993.

* Correspondence should be sent to Dr N. Cimolai. Microbiology, Department of Pathology, British Columbia's Children's Hospital, 4480 Oak Street, Vancouver, British Columbia, Canada V6H 3V4. include infection of human volunteers, assessment of seroconversion and placebo-controlled treatment studies.

The present studies were initiated to improve our knowledge of the human humoral immune response to $S$. equisimilis and to a range of other $\beta$-haemolytic streptococci, by immunoblotting.

\section{Materials and methods}

\section{Bacterial isolates}

The six strains of $S$. equisimilis investigated were isolated from the throats of children with acute pharyngitis., ${ }^{4,5}$ Strain no. 6198 was used for most of the immunoblotting studies as identical SDS-PAGE patterns had been found in $c .50 \%$ of $S$. equisimilis strains that were obtained from human origin. This strain and the other $S$. equisimilis strains were characterised by Lancefield grouping (group C), carbohydrate fermentation, the Voges-Proskauer test and $\beta$ D-glucuronidase activity. ${ }^{7}$ Strains of $S$. pyogenes (group A), large colony human biotype group G streptococci, group $C$ and G streptococci of the " $S$. anginosus-milleri group" and other streptococci were acquired either from our previous studies or from clinical specimens received in the routine diagnostic microbiology laboratory at British Columbia's Children's Hospital. These isolates were also acquired from the throats of symptomatic or asymptomatic children and represent wild-type isolates. Bacteria had been stored at $-70^{\circ} \mathrm{C}$ and were previously passaged only 




Fig. 1. IgG immunoblotting of $S$. equisimilis strain no. 6198 with various samples of human adult donor serum (lanes 1-8). Antigens of 46, 66 and $105 \mathrm{kDa}$ are predominantly recognised. Lane 9 confirms the lack of non-specific Fc binding of the anti-IgG conjugate. The markers on the left indicate mol.-wt standards.

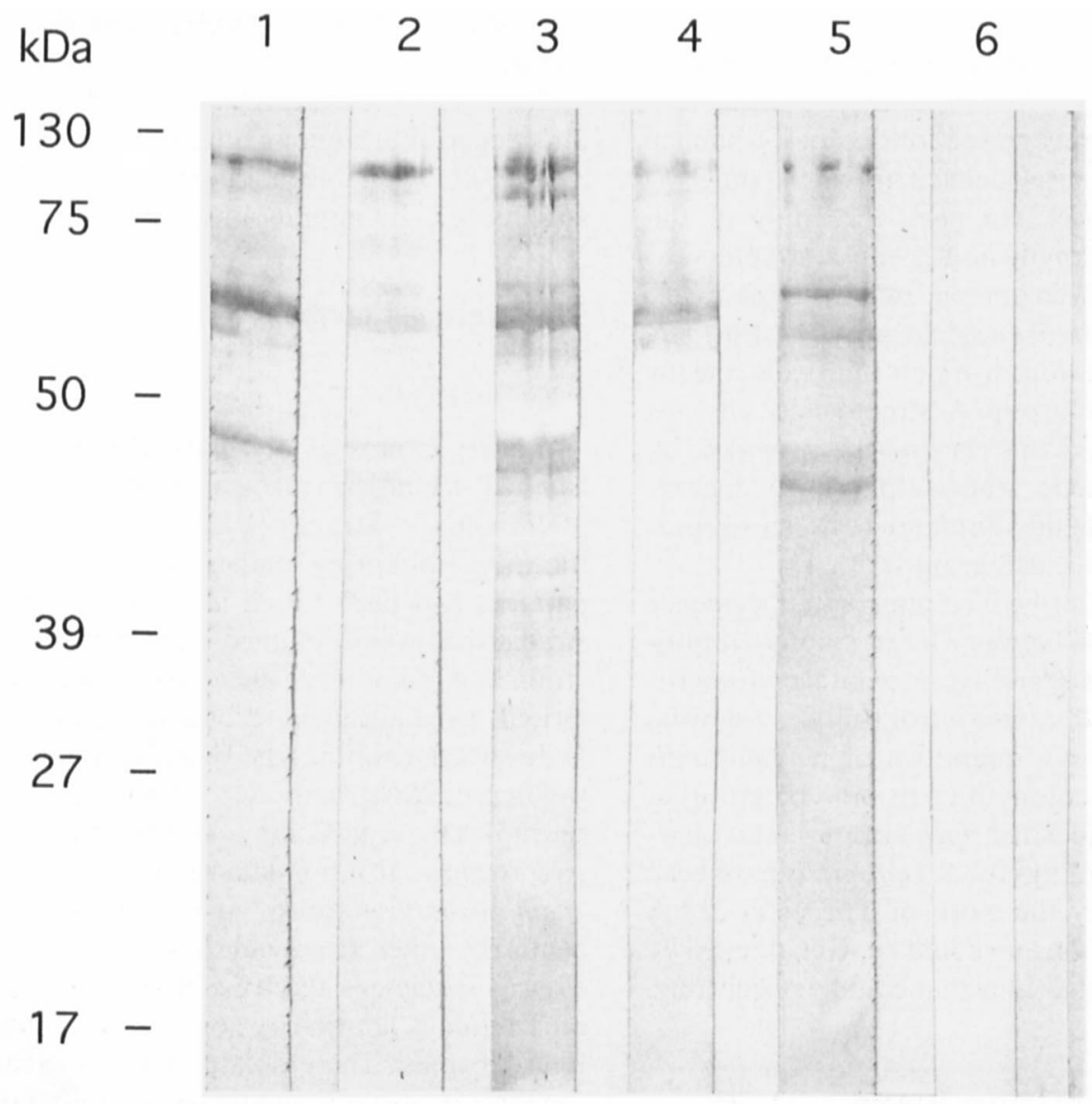

Fig. 2. IgA (heavy chain) immunoblotting of S. equisimilis strain no. 6198 with various samples of human adult donor serum (lanes 1-5) Antigens of 46,66 and $105 \mathrm{kDa}$ are predominantly recognised. Lane 6 confirms the lack of non-specific $F c$ binding of the anti-IgA conjugate. Mol.-wt markers as for fig. 1. 




Fig. 3. IgG immunoblotting of five wild-type S. equisimilis strains with a single human serum. These strains are known to differ in their SDSPAGE profiles. Mol.-wt markers as for fig. 1.

three times or less. We chose to use wild-type strains for our studies because of the uncertainty of passage and the potential for change in virulence factors of highly-passaged type strains.

\section{Sera and respiratory specimens}

Serum was collected from 20 healthy volunteer adults and sputum from 20 adolescent paediatric inpatients with cystic fibrosis. To reduce the chance of salivary contamination to a minimum, all sputum samples were examined microscopically for the presence of squamous epithelial cells. Samples containing more than a few epithelial cells by low power microscopy were discarded. Sputum was centrifuged at $1500 \mathrm{~g}$ and the supernates were stored at $-70^{\circ} \mathrm{C}$ until required for $\operatorname{IgA}$ immunoblotting studies. Serum samples were stored under the same conditions.

\section{Gel electrophoresis and Western transfer}

Bacteria were inoculated from the frozen state on to sheep blood $5 \%$ agar media (Columbia base) and then incubated overnight at $37^{\circ} \mathrm{C}$. The purity of cultures was confirmed and strains were inoculated into ToddHewitt broth and incubated aerobically at $35^{\circ} \mathrm{C}$ for 18-24 $\mathrm{h}$. The biomass was centrifuged, washed three times and then suspended in phosphate-buffered saline (PBS; pH 7.2). These suspensions were standardised by estimating the whole-cell protein equivalent by the Bradford method (BioRad). Initial suspensions were diluted or concentrated to achieve a protein equivalent of $c .700 \mu \mathrm{g} / \mathrm{ml}$. This preparation then served as the stock for subsequent SDS-PAGE. Samples of this stock were boiled in reducing sample buffer $(1: 4$ ratio; $5 \times$ sample buffer: $0.25 \mathrm{M}$ Tris, glycerol $50 \%$, SDS $10 \%, \beta$-mercaptoethanol $5 \%$ ) and then subjected to conventional one-dimensional SDS-PAGE which included $3 \%$ stacking and $10 \%$ running gels. ${ }^{8}$ After the electrophoresis was completed, the resolved products were transferred to $0.45-\mu \mathrm{m}$ nitrocellulose in an electrophoresis unit (Hoefer Scientific Instruments, CA, USA) for 30 min with a direct current of $1.5 \mathrm{~A}$. Pre-stained mol.-wt markers (BioRad) were used for the determination of approximate antigen migration rates and corresponding mol. wts. 


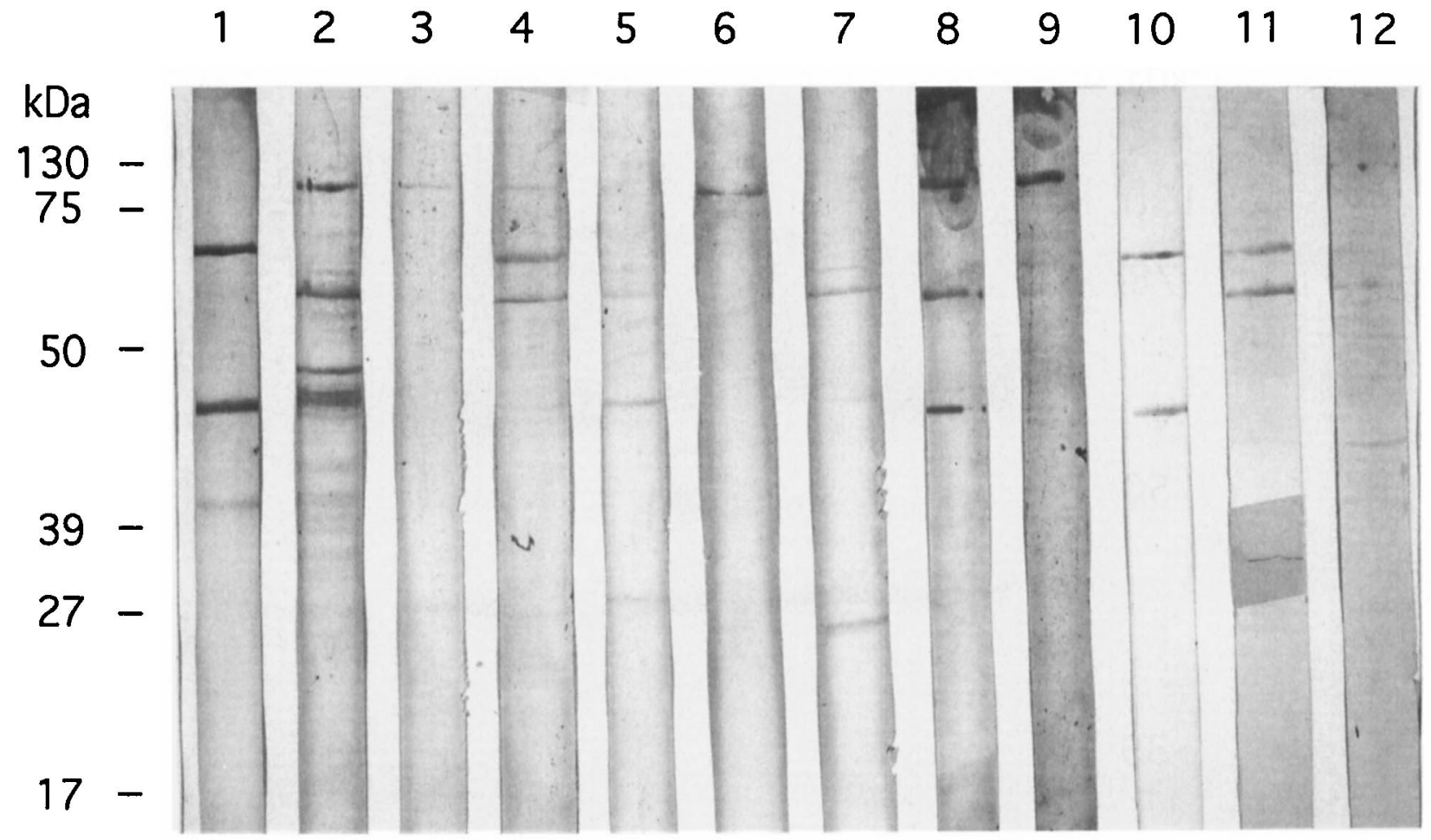

Fig. 4. IgA (heavy chain) and secretory IgA (J chain) immunoblotting of $S$. equisimilis strain no. 6198 with sputum samples as the antibody source: lanes $1-9$ include examples of immunoblot patterns for IgA (heavy chain) reactivity; 10-12 represent secretory IgA patterns; 10, 11 and 12 were with the same sputum samples as 1,4 and 9 , respectively. Note the recognition of a $73-\mathrm{kDa}$ antigen in lanes $1,4,10$ and 11 . Mol.-wt markers as for fig. 1 .

\section{Immunoblotting}

To assess the presence of antibody to the various bacteria, strips containing the profile of polypeptides for each bacterium were cut from the nitrocellulose. The nitrocellulose strips were blocked with a mixture of skimmed milk $1 \%$ and bovine serum albumin $1 \%$ in PBS. This stage was crucial because it prevented endogenous streptococcal Fc-binding fragments from interfering with bacterium-specific antibody recognition patterns. ${ }^{9}$ Strips were incubated overnight at $4^{\circ} \mathrm{C}$ or for $1 \mathrm{~h}$ at $35^{\circ} \mathrm{C}$. Immunoblotting was performed to assess the presence of $\operatorname{IgG}, \operatorname{IgA}$ or secretory $\operatorname{IgA}$ (sIgA) antibodies from either serum or sputum samples. For serum IgG or IgA assays, the human sera were diluted 1 in 100 in skimmed milk $1 \%$ in PBS and then incubated for $1 \mathrm{~h}$ at $35^{\circ} \mathrm{C}$. The nitrocellulose support was washed three times with PBS and then incubated with a 1 in 500 dilution (in skimmed milk $1 \%$ in PBS) of goat anti-human IgG or IgA (heavy chain)-alkaline phosphatase-conjugated antibody (Jackson Immunoresearch, PA, USA). 1-Nitroblue tetrazolium chloride and 5-bromo-4-chloro-3-indolyl phosphate substrates were chosen for the alkaline phosphatase. For the detection of antibody in sputum, a 1 in 50 dilution of sputum supernate replaced the serum analogue in the preceding assay. Whereas the anti-IgA (heavy chain) was used as before in the remainder of the assay, the detection of $\operatorname{sgA}$ was accomplished with murine monoclonal antibody
(MAb) directed against the J chain of IgA (MAb GA1 ; ICN Biomedicals, CA, USA). For the latter, a 1 in 250 dilution (in skimmed milk $1 \%$ in PBS) of the MAb was used after incubation of the sputum supernate with the nitrocellulose strip. Goat anti-mouse IgGperoxidase conjugate (dilution 1 in 500 ; BioRad) was used as the final antibody and 4-chloronaphthol served as the peroxidase substrate. For anti-vimentin immunoblotting, both murine MAb (clone V9; Dakopatts, Denmark) and rabbit polyclonal antibody (ICN Biomedicals) were used. The MAb was diluted 1 in 300 and the procedure followed essentially the same protocol as used for the $\mathbf{J}$ chain $\mathrm{MAb}$. Rabbit polyclonal anti-vimentin antibody was diluted 1 in 600 and then detected with goat anti-rabbit IgGhorseradish peroxidase ( 1 in 1000 dilution; BioRad).

\section{Affinity purification and re-blotting}

To determine whether the $66-\mathrm{kDa}$ antigen of $S$. equisimilis shared immunoreactive epitopes with the $60-\mathrm{kDa}$ antigen of $S$. pyogenes, the following experiment was performed. The immunoreactive polypeptides of $S$. equisimilis were localised by $\operatorname{IgG}$ immunoblotting. Unused portions of contiguous nitrocellulose that contained the $66-\mathrm{kDa}$ antigen were excised, blocked with skimmed milk $1 \%$ in PBS, and then incubated in 1 in 100 dilutions of human serum for $2 \mathrm{~h}$ at $35^{\circ} \mathrm{C}$. After washing five times in PBS, the polyclonal human immunoglobulin was eluted from 
$\begin{array}{llll}\text { A } & B & C & \text { D }\end{array}$

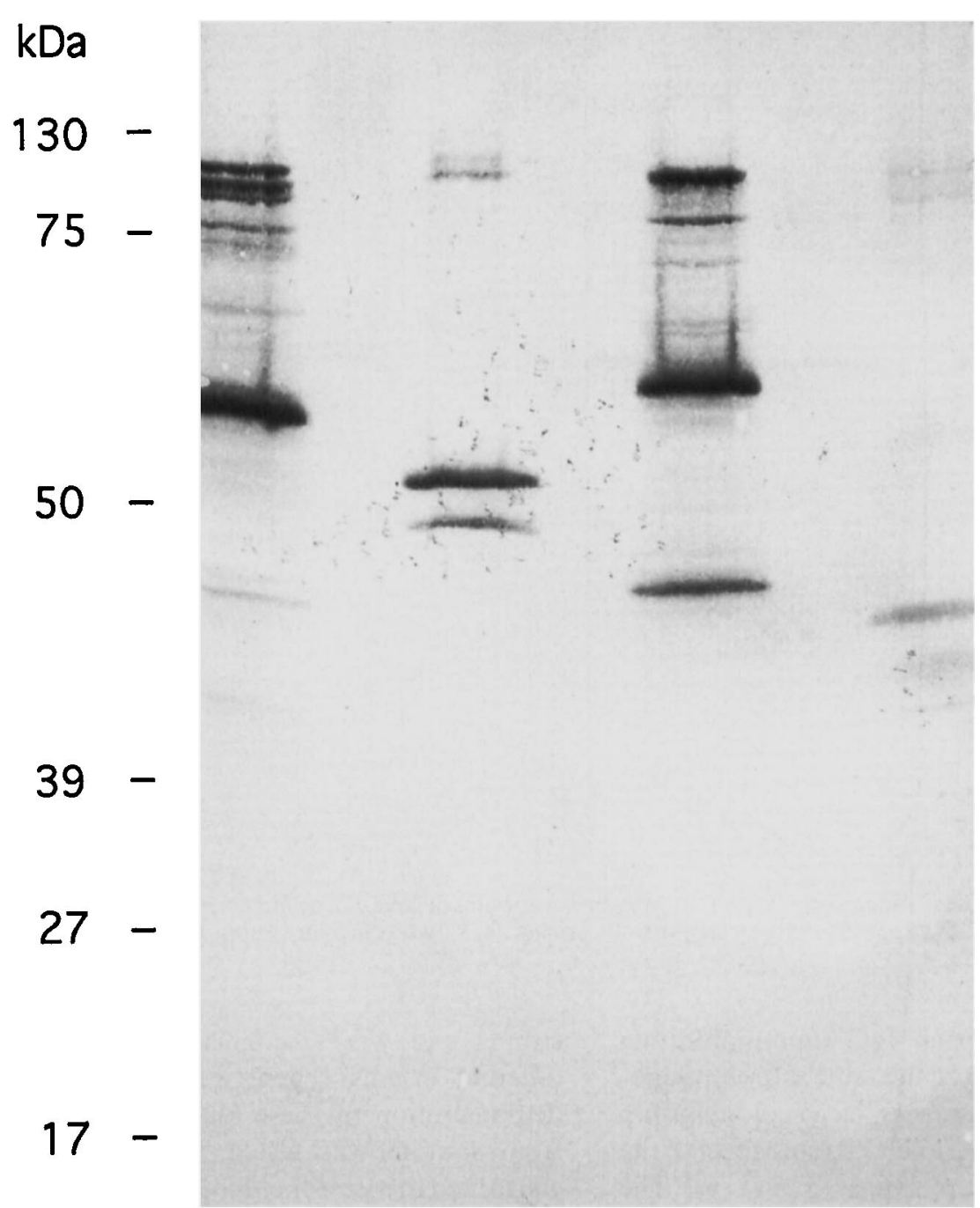

Fig. 5. IgG immunoblotting of various streptococci: lanes A-D, S. pyogenes, group B streptococcus, S. equisimilis strain no. 6198 and Enterococcus faecalis. Note the variation of S. equisimilis $(66 \mathrm{kDa})$ and $S$. pyogenes $(60 \mathrm{kDa})$ antigens. Mol.-wt markers as for fig. 1.

the nitrocellulose by exposure to $0.2 \mathrm{M}$ glycine- $\mathrm{HCl}$ $(\mathrm{pH} 28)$ for $15 \mathrm{~min}$ at $4^{\circ} \mathrm{C}$. After the elution, the antibody in solution was dialysed overnight against PBS (pH 7.2). The dialysed eluate (undiluted) was then used as antibody in immunoblotting with the $S$. pyogenes substrate.

\section{Results}

Human serum IgG and IgA identified three main antigens of $S$. equisimilis strain no. 6198 with migration rates equivalent to mol. wts of c. 46, 66 and $105 \mathrm{kDa}$ (figs. 1 and 2). There were minor variations of immunoblot patterns with different human sera and antigens $<46 \mathrm{kDa}$ were rarely recognised. Although c. $50 \%$ of $S$. equisimilis strains of human origin ( 30 wild-type, epidemiologically unlinked isolates) that we have studied yielded SDS-PAGE profiles identical to strain no. 6198, at least seven other SDS-PAGE patterns could be defined by major or minor vari- ations. Sera were used to probe immunodominant antigens among other $S$. equisimilis strains that had SDS-PAGE patterns different from that of strain no. 6198. Similar antigens were immunodominant (fig. 3). These strains represent c. $80 \%$ of SDS-PAGE patterns among our wild-type $S$. equisimilis isolates. Control immunoblots, with bacterial growth media as the substrate for antibody recognition, were negative.

Half of the sputum samples (10 of 20) had specific IgA to $S$. equisimilis that could be demonstrated with anti-heavy chain conjugates. Examples with stronger resolution are illustrated in fig. 4 . The main antigens recognised were similar to those detected with serum samples. Among the 10 sputum samples that contained anti-S. equisimilis IgA (heavy chain), only four had evidence of anti-S. equisimilis SIgA. In addition to variable recognition of the three dominant antigens, an antigen of $73 \mathrm{kDa}$ was occasionally recognised by IgA (heavy chain) or sIgA from sputum. This antigen was not prominently highlighted in any of the serum immunoblotting. 


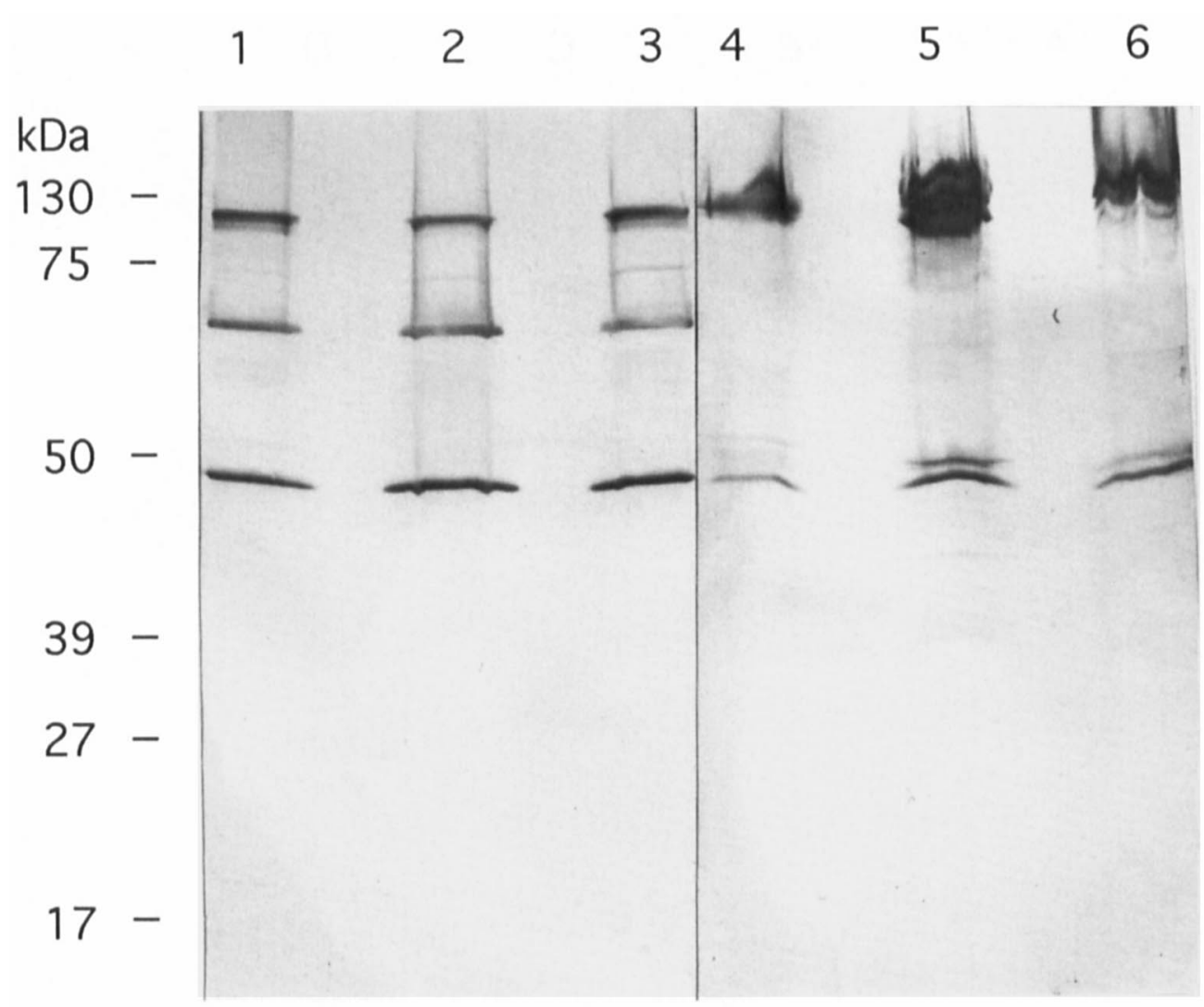

Fig. 6. IgG immunoblotting of various streptococci: lanes 1-3, three different strains of large colony human biotype group G streptococci that have been demonstrated previously to possess different SDS-PAGE profiles; 4-6, three different strains of group C " $S$. anginosus-milleri group". Mol.-wt markers as for fig. 1.

Further studies with serum IgG immunoblotting identified what appeared to be the same three immunodominant antigens in $S$. pyogenes, large colony group $\mathrm{G}$ streptococci, and group $\mathrm{C}$ and $\mathrm{G}$ streptococci of the " $S$. anginosus-milleri group" (figs. 5 and 6). The patterns obtained with group B streptococci and enterococci showed that these did not possess the 46-, 66- or 105-kDa antigens.

Although the presence of the triplet fingerprint of immunodominant antigens among the different streptococci was most striking, it was apparent that antigens putatively homologous between species did not have identical migration rates, e.g., the $66-\mathrm{kDa}$ antigen of $S$. equisimilis appeared to have a homologue of $60 \mathrm{kDa}$ in S. pyogenes (fig. 5). Minor variation of the same antigen was also noted among $S$. equisimilis strains (fig. 3). When polyclonal antibody was eluted from the $66-\mathrm{kDa}$ antigen of $S$. equisimilis, it was found to bind to the $60-\mathrm{kDa}$ antigen of $S$. pyogenes, thereby confirming the suspicion that these antigens shared epitopes.

Both monoclonal and polyclonal anti-vimentin antibodies recognised the 46- and $66-\mathrm{kDa}$ antigens of $S$. equisimilis (not shown). Controls for growth medium components were uniformly negative.

\section{Discussion}

The three immunodominant antigens described were consistently present among different $S$. equisimilis strains and were recognised by human serum of different origins. There was some variation in the demonstration of these antigens when $\operatorname{IgA}$ or $\operatorname{sIgA}$ from sputum was tested, but further study seems warranted to assess their potential role in pathogenesis and in the development of immunodiagnostic assays. However, the frequency and degree of their recognition by the humoral immune responses implies that qualitative immunoblotting assays alone will be insufficient. Nevertheless, it is conceivable that quantitative assays that examine humoral responses to these antigens may be of value. In addition to the triplet of commonly recognised antigens, studies of IgA in sputum demonstrated a $73-\mathrm{kDa}$ antigen. It is remarkable that serum IgG and IgA did not highlight this same antigen. Immunoblotting has been employed to investigate the association of various streptococci and guttate psoriasis, ${ }^{10}$ but the immunodominant antigens described here were not reported. Rather, the latter paper proposed that differences in the immunoblotting response among patient groups could be defined on the basis of both the number and intensity of staining of the antigens recognised.

The presence of the triplet of immunodominant antigens among strains of $S$. equisimilis and large colony group $\mathrm{G}$ streptococci is not surprising, as they are probably homogeneous. ${ }^{2,3,7,9}$ The fact that the antigens were also found in $S$. pyogenes could be related to all three species being primary pharyngeal pathogens with the ability to cause infections of 
sufficient severity to stimulate systemic and mucosal antibody responses to these antigens. Previous studies have proposed homology between streptococci of Lancefield groups A, C and $\mathrm{G}$ by the demonstration of common M-like proteins. ${ }^{711,12}$ The association of the " $S$. anginous-milleri group" with symptomatic pharyngitis is in doubt ${ }^{5}$ and it is known that mucosal colonisation is common and increases with age. ${ }^{4}$ Therefore, the presence of the triplet of immunodominant antigens in this group of streptococci detracts somewhat from the hypothesis that these antigens are related to pathogenesis.

Several studies have demonstrated evidence of immunological cross-reactivity between $S$. pyogenes $\mathrm{M}$ proteins, vimentin, cardiac antigens and the $S$. pyogenes $60-\mathrm{kDa}$ antigen. ${ }^{13-17}$ Some of these studies have proposed that these immunological similarities have significant implications for the understanding of both rheumatic fever and enteroviral myocarditis. Whereas it is believed that the $\mathrm{M}$ proteins of $S$. pyogenes are prime candidates for stimulating an auto-antibody response that may be the fundamental pathology in acute rheumatic fever, the $60-\mathrm{kDa}$ antigen also crossreacts with heart tissue. Our results seem to suggest that the $S$. pyogenes $60-\mathrm{kDa}$ antigen and the $S$. equisimilis $66-\mathrm{kDa}$ antigen share epitopes, and that this antigen in addition to the $46-\mathrm{kDa}$ antigen shares epitopes with vimentin. The presence of these antigens in less pathogenic but commonly found streptococci indicates that the role of such antigens in the pathogenesis of rheumatic heart disease may be less than previously believed. Ayakawa et al. ${ }^{14}$ have also used anti-S. pyogenes MAbs to identify a $67-\mathrm{kDa}$ antigen in $S$. mutans; the latter organism rivals bacteria of the "S. anginosus-milleri group" in frequency of human oropharyngeal colonisation. A function remains to be defined for the $60-\mathrm{kDa}$ antigen of $S$. pyogenes, although proteins of similar mol. wt have included protein kinases, actin-like filaments and streptolysin O. ${ }^{18-20}$

The value of the immunodominant antigens described here for serodiagnostic purposes remains to be fully investigated. Furthermore, the complex antigenic relationships of the $\beta$-haemolytic streptococci that have been demonstrated warrant further study in the context of auto-antibody formation and the pathogenesis of post-infectious sequelae of streptococcal infections.

\section{References}

1. Cimolai N, Elford RW, Bryan L, Anand C, Berger P. Do the beta-hemolytic non-group A streptococci cause pharyngitis? Rev Infect Dis 1988; 10: $587-601$.

2. Farrow JAE, Collins MD. Taxonomic studies on streptococci of serologic groups $\mathrm{C}, \mathrm{G}$, and $\mathrm{L}$ and possibly related taxa. Systemic Appl Microbiol 1984; 5: 483-493.

3. Kilpper-Balz R, Schleifer KH. Nucleic acid hybridization and cell wall composition studies of pyogenic streptococci. FEMS Microbiol Lett 1984; 24: 355-364.

4. Cimolai N, MacCulloch L, Damm S. The epidemiology of betahemolytic non-group A streptococci isolated from the throats of children over a one-year period. Epidemiol Infect 1990; 104: 119-126.

5. Cimolai N, Morrison BJ, MacCulloch L, Smith DF, Hlady J. Beta-hemolytic non-group A streptococci and pharyngitis: a case-control study. Eur J Pediatr 1991; 150: 776-779.

6. Turner JC, Fox A, Fox K et al. Role of group C beta-hemolytic streptococci in pharyngitis: epidemiologic study of clinical features associated with isolation of group $\mathrm{C}$ streptococci. $J$ Clin Microbiol 1993; 31: 808-811.

7. Cimolai N, Mah D. Beta-D-glucuronidase activity assay for rapid differentiation of species within beta-hemolytic group C and G streptococci. J Clin Pathol 1991; 44: 824-825.

8. Laemmli UK. Cleavage of structural proteins during the assembly of the head of bacteriophage T4. Nature 1970; 227: $680-685$.

9. Cimolai N, Cheong ACH. Differentiation of species in human beta-hemolytic group $\mathrm{G}$ streptococci using immunoglobulin Fc fragment receptor. $J$ Clin Puthol 1992; 45: 232-234.

10. Wilson AGM, Clark I, Heard SR, Munro DD, Kirby JDT. Immunoblotting of streptococcal antigens in guttate psoriasis. Br J Dermatol 1993; 128: 151-158.

11. Fischetti VA. Streptococcal M protein: molecular design and biological behavior. Clin Microbiol Rev 1989; 2: 285-314.

12. Simpson WJ, Musser JM, Cleary PP. Evidence consistent with horizontal transfer of the gene (emm 12) encoding serotype M12 protein between group A and group $G$ pathogenic streptococci. Infect Immun 1992; 60: 1890-1893.

13. Kraus W, Ohyama K, Snyder DS, Beachey EH. Autoimmune sequence of streptococcal $M$ protein shared with the intermediate filament protein, vimentin. $J$ Exp Med 1989; 169: 481-492.

14. Ayakawa GY, Bleiweis AS, Crowley PJ, Cunningham MW. Heart cross-reactive antigens of mutans streptococci share epitopes with group A streptococci and myosin. J Immunol $1988 ; 140: 253-257$.

15. Cunningham MW, Antone SM, Gulizia JM, McManus BM, Fischetti VA, Gauntt CJ. Cytotoxic and viral neutralizing antibodies crossreact with streptococcal M protein, enteroviruses, and human cardiac myosin. Proc Natl Acad Sci USA 1992; 89: 1320-1324.

16. Gulizia JM, Cunningham MW, McManus BM. Immunoreactivity of anti-streptococcal monoclonal antibodies to human heart valves. Evidence for multiple cross-reactive epitopes. Am J Pathol 1991; 138 : 285-301.

17. Barnett LA, Cunningham MWA. A new heart-cross-reactive antigen in Streptococcus pyogenes is not M protein. $J$ Infect Dis 1990 ; 162: 875-882.

18. Chiang TM, Reizer J, Beachey EH. Serine and tyrosine protein kinase activities in Streptococcus pyogenes. Phosphorylation of native and synthetic peptides of streptococcal $M$ proteins. J Biol Chem 1989; 264: 2957-2962.

19. Barnett LA, Cunningham MW. Evidence for actinlike proteins in an M protein-negative strain of Streptococcus pyogenes. Infect Immun 1992; 60: 3932-3936.

20. Canalias F, Viver J, Beleta J, Gonzalez-Sastre F, Javier-Gella F. Purification and characterization of streptolysin $O$ from Streptococcus pyogenes. Int $J$ Biochem 1992; 24: 1073-1079. 